\title{
Evaluating individual performance for clinical governance
}

\author{
Gill Wakley
}

\section{Individual competence}

Doctors "must be committed to lifelong learning and be responsible for maintaining the medical knowledge and clinical and team skills necessary for the provision of quality care". ${ }^{1}$ The Nursing and Midwifery Council (NMC) also stresses the importance of lifelong learning. The NMC recognises that health care is an area of constant change that necessitates a dynamic approach to learning. In order to develop and maintain competence, nurses are required to "demonstrate responsibility for your own learning through the development of a portfolio ... and to be able to recognise when further learning and development may be required". 2

Describing your roles and responsibilities will help you to define what your competence should be now, or what competence you are hoping to attain, for example, as a practitioner with a special interest $(\mathrm{PwSI}){ }^{3}$ You might compare your current competency with that required by the Faculty of Family Planning and Reproductive Health Care. ${ }^{4}$ You will need to describe the standards expected in the range of tasks and roles you undertake, and reference the source of standard setting, for example, from the Faculty 4 or elsewhere. 5

To perform consistently well requires efficient systems and sufficient resources. You will need adequate numbers of other competent doctors or staff and a readily available infrastructure such as diagnostic and treatment resources. ${ }^{6}$

\section{Identifying learning needs}

Use several methods to demonstrate your competence and identify your learning needs (and gaps in your service development or delivery) so that you validate the findings of one method by another. No one method will give you reliable information about your knowledge, skills, attitudes or everyday service. It is particularly difficult to determine what it is you 'don't know you don't know' by yourself, yet it is vital that you identify and rectify those deficiencies.

Find out what you are achieving by:

- asking patients, users and non-users of your service

- comparing your performance against best practice or that of peers

- comparing your performance against objectives in business plans or national directives

- asking colleagues from different disciplines about how your work interfaces with theirs.

\section{Documenting your competence}

You may decide to use a few selected methods to gather baseline evidence of your performance, focused on your specific area of expertise. You may target other topics or areas at the same time that are relevant to the various sections of the General Medical Council's booklet, Good Medical Practice ${ }^{7}$ (Box 1).

J Fam Plann Reprod Health Care 2005; 31(1): 46-48

(Accepted 7 October 2004)

Staffordshire University, Stafford, UK

Gill Wakley, MD, MFFP, Visiting Professor in Primary Care

Development and Freelance General Practitioner

Correspondence to: Professor Gill Wakley.

E-mail: gillwak@aol.com
Box 1: The duties and responsibilities of doctors ${ }^{7}$

1. Good professional practice. This relates to clinical care, keeping records (including writing reports and keeping colleagues informed), access and availability, treatment in emergencies and making effective use of resources.

2. Maintaining good medical practice. This includes keeping up to date and maintaining your performance.

3. Relationships with patients. This encompasses providing information about your services, maintaining trust, avoiding discrimination and prejudice against patients, relating well to patients and apologising if things go wrong.

4. Working with colleagues. This relates to working with colleagues, working in teams, referring patients and accepting posts.

5. Teaching and training, appraising and assessing. You may be in a position to teach or train colleagues or students, and appraise or assess peers, employees or students.

6. Probity includes providing true information about your services, honesty in financial and commercial dealings, providing references.

7. Health can include how you overcome or compensate for health problems in yourself, or help with or address health problems in other doctors.

Research and management responsibilities may also be included.

For this type of combined assessment, you might use several methods such as:

- constructive feedback from peers or patients

- $360^{\circ}$ feedback

- self-assessment, or review by others, using a rating scale to assess your skills and attitudes

- comparison with protocols and guidelines for checking how well procedures are followed

- audits of various types and applications including significant event audit

- a SWOT (strengths, weaknesses, opportunities and threats) or SCOT (strengths, challenges, opportunities and threats) analysis

- eliciting patient views such as satisfaction surveys

- reading and reflecting

- educational review.

Several of these methods will also be useful for identifying service development needs - you can look at the gaps identified from both the personal and service perspectives at the same time using the same method. These methods are described in more detail in Demonstrating Your Clinical Competence in Women's Health. ${ }^{8}$

\section{Audit}

Audit as a tool seems to be widely misunderstood. Research is determining what should be done but audit finds out if you have done the right things and how well you have done them. The five essential steps of the audit cycle are to:

1. Define the domain being examined and the criteria and standards you are trying to achieve.

2. Measure your current performance of how well you are providing care or services in an objective way.

3 Compare your performance against criteria and standards.

4. Identify the need for change - to performance, adjustment of criteria or standards, resources or available data.

5. Make any required changes as necessary and re-audit later. 
All too often the domain is not clear and no standards are set (Step 1), the collection of data is descriptive (Steps 2 and 3), there is no mechanism for using the data as an instrument to make changes (Step 4) and no check to establish whether any change has occurred (Step 5). A useful summary of what to do before starting audit is provided below (Box 2).

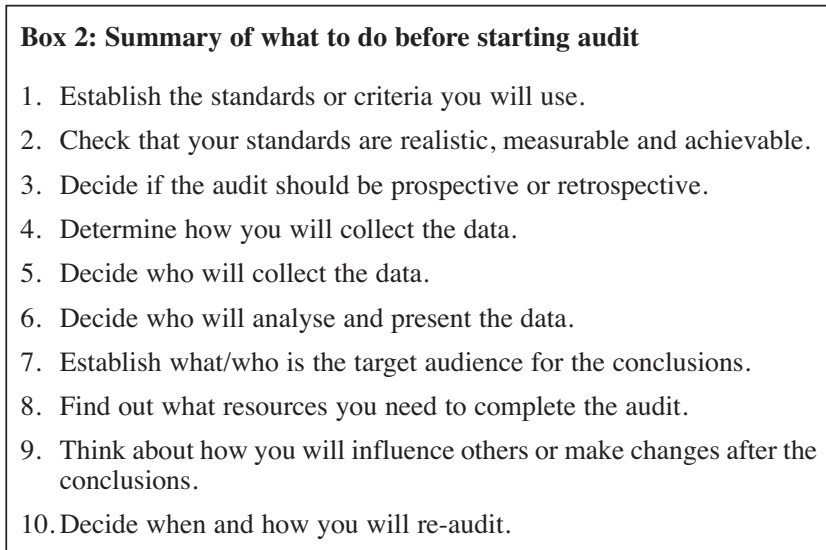

You might like to look back at some previously published audits on family planning ${ }^{9-11}$ and determine for yourself whether all the aspects listed above were fulfilled and, if not, how you might have done better.

Performance or practice is often divided into three aspects:

- Structure: audits might concern resources such as equipment, premises, skills, number of people identified with a condition or being given a treatment, etc. (Box 3).

\section{Box 3: Example of an audit of structure}

A patient asks to be seen that evening for provision of contraception. You are running late and already have several extra patients waiting to be seen. You suggest she is booked in for the next clinic instead. She attends too late for emergency oral contraception. You decide to review the way in which urgent requests are managed.

- Process: focuses on what is done to the patient; for instance, clinical protocols and guidelines (Box 4).

Box 4: Auditing process
It is recommended that you should record the blood pressure of all
women before starting on oral contraceptives and that you should check
it regularly thereafter. 12,13 You could set standards together with your
colleagues: e.g. $100 \%$ of women have their blood pressure measured
before combined oral contraceptives are prescribed and $90 \%$ of those on
combined oral contraception have a recorded blood pressure level in the
last 12 months. Then audit your records for compliance and, if your
results are not satisfactory, take action before re-auditing.

- Outcomes: considers the impact of care or services on the patient and might include patient satisfaction, health gains and effectiveness of care or services (Box 5).

Box 5: Auditing outcome

It has been shown that the incidence of complications with intrauterine devices is lower when clinicians fit them regularly. ${ }^{14}$ Compare your rates with those of published outcome studies and determine if you need to increase your experience or revise your techniques.

You might look at aspects of quality of the structure, process and outcome of the delivery of any clinical field focusing on access, equity of care between different groups in the population, efficiency, economy, effectiveness for individual patients, etc. ${ }^{6}$

\section{Sampling for audit}

Audit is not like research where you are hoping that your results will be generalisable to a wider population than the one examined. You are simply looking at this local setting but it is still important not to look at an unrepresentative sample. If the target population is less than 100 people, you might look at all of them. Look at a guide on audit for advice on choosing your sample size. ${ }^{15}$ In random sampling using a random number table everyone has an equal chance of being selected. Systematic sampling requires you to select every $n$th record, for example, if the target group is 200 and the sample size 50, you would select every fourth record (i.e. 200 divided by 50 ). Consecutive samples are often convenient, e.g. the next 50 patients with that complaint, but make sure you have a large enough sample so that you do not make changes based on poor evidence.

\section{Types of audit}

Some variations on audit include:

- Case note analysis. This gives an insight into your current practice. It might be a retrospective review of a random selection of notes, or a prospective survey of consecutive patients with a particular problem.

- Peer review. Compare an area of practice with other individual professionals or managers or compare clinical teams. An independent body might compare all clinics or practices in one area. Feedback may be arranged to protect participants' identities so that only the individual person or practice knows their own identity, the rest being anonymised, for example, by giving each person a number. Where there is mutual trust and an open learning culture, peer review does not need to be anonymised and everyone can learn together about making improvements in practice.

- Criteria-based audit. This compares clinical practice with specific standards, guidelines or protocols. Reaudit of changes should demonstrate improvements in the quality of patient care.

- External audit. Prescribing advisers or managers can supply information about indicators of performance for audit. Visits from external bodies expose organisation to external audit.

- Tracer criteria. Assessing the quality of care of a 'tracer' condition may be used to represent the quality of care of other similar conditions or more complex problems. Tracer criteria should be easily defined and measured. For instance, you might audit the follow-up after you have instigated an investigation such as testing for chlamydia and generalise from your audit results to your likely performance with other infections.

\section{Significant event audit}

Think of an incident where a patient or you experienced an adverse event. This might be an unplanned pregnancy, a side effect from prescribed medication or treatment, a violent attack on a member of staff, or an angry outburst in public by you or a work colleague. You can review the case and reflect on the sequence of events that led to that critical event occurring (Box 6). It is likely that there were a multitude of factors leading up to that significant event. You should take the case to a multidisciplinary meeting to reflect and analyse what were the triggers, causes and consequences of the event. Complete the significant event audit cycle by planning what individuals or the practice as a whole might do to avoid a similar event happening in future. This might include undertaking further learning and/or making appropriate changes to how you and others work. ${ }^{16}$ 
Box 6: Steps of a significant event audit

1. Describe who was involved, what time of day, what task/activity, the context and any other relevant information.

2. Reflect on the effects of the event on the participants and the professionals involved.

3. Discuss the reasons for the event or situation arising with other colleagues, review case notes or other records.

4. Decide how you or others might have behaved differently. Describe your options for how the procedures at work might be changed to minimise or eliminate the event from recurring.

5. Plan changes that are needed, how they will be implemented, who will be responsible for what and when, what further training or resources are required. Then carry out the changes.

6. Re-audit later to see whether changes to procedures or new knowledge and skills are having the desired effects. Give feedback to all involved.

\section{Challenges}

There are undoubted difficulties in establishing how well we are doing - demonstrating our competence as individual clinicians for clinical governance.

- The lack of evidence-based guidelines is gradually being addressed, but the evidence for much of what we do simply does not exist. Standard practice is just what was thought to be a good idea at the time of introduction, for example, leaving 7 days between one packet of combined oral contraceptives and the next.

- Many of the outcomes of contraceptive care are dependent on process variables, such as teaching how to use contraception or counselling about behaviour changes, and are difficult to measure.

- How do you measure a 'non-event'? Measuring conception rates is a surrogate marker for contraceptive effectiveness and measuring 'avoidance of infection' is even more problematical. Measuring knowledge or skills levels may be more manageable - but does this translate into effective action?

- Many activities might be better examined as research projects - but applying for ethical committee approval for 'research' is now so complicated 17 that they are often turned inappropriately into audit procedures, even when no standard exists against which to measure them.

- Audit standards are often inappropriate. For example, it sounds a good idea to set as a standard ' $90 \%$ of patients who receive emergency contraception (EC) and are more than 7 days late for their period should have a pregnancy test'. Where will they have the test? What if they do not return to the place where they obtained the $\mathrm{EC}$, etc.?
- Record data from a sufficient number of patients to convince yourself, or your colleagues, that changes are necessary. Too few and you may conclude that you 'usually' do better.

- Ensure that you do not spend so much time recording and measuring that you do not have time to do the essential work properly.

The types of activity for which individual clinicians or staff are responsible are listed in the previous article in this series. ${ }^{18}$ The next article in this series looks at service provision and clinical governance requirements.

Statements on funding and competing interests

Funding. None identified.

Competing interests. None identified.

References

Medical Professionalism Project. Medical professionalism in the new millennium: a physicians' charter. Lancet 2002; 359: 520-522.

2 Nursing and Midwifery Council (NMC). Supporting Nurses and Midwives Through Lifelong Learning. London, UK: NMC, 2002.

3 http://www.gpwsi.org.

4 http://www.ffprhc.org.uk

5 http://www.agum.org.uk/filingcab/standards_under25_0602.pdf.

6 Fraser SW, Greenhalgh T. Coping with complexity: educating for capability. BMJ 2001; 323: 799-802.

7 General Medical Council (GMC). Good Medical Practice. London, UK: GMC, 2001

8 Campbell P, Wakley G, Chambers R, Jenkins J. Demonstrating Your Clinical Competence in Women's Health (Primary Care Nursing Series). Oxford, UK: Radcliffe Medical Press, 2004

9 Murty J, Barron A, Searle ES. Auditing the introduction of a new product to a family planning service. Br J Fam Plann 1998; 24 $24-25$.

10 Smith A. Staff awareness, acceptance and compliance with locally developed guidelines within a community family planning service. J Fam Plann Reprod Health Care 2002; 28: 39-40.

11 Siddiqui F, Kirkman R, Chandiok A. Re-audit of referral compliance of chlamydia-positive women from a family planning clinic. J Fam Plann Reprod Health Care 2004; 30: 86-87.

12 Hannaford P, Webb A. Evidence-guided prescribing of oral contraceptives. Contraception 1996; 54: 125.

13 Faculty of Family Planning and Reproductive Health Care Clinica Effectiveness Unit. FFPRHC Guidance (October 2003). Firs prescription of combined oral contraception. J Fam Plann Reprod Health Care 2003; 29(4): 209-223.

14 Rowe PJ. Research on Intrauterine Devices. World Health Organization (WHO) Special Programme of Research, Development and Research Training in Human Reproduction. Annual Technical Report. Geneva, Switzerland: WHO, 1992

15 National Health Service, National Institute for Clinical Excellence, Commission for Health Improvement, Royal College of Nursing Principles for Best Practice in Clinical Audit. Oxford, UK: Radcliffe Medical Press, 2002.

16 Ingram S, Randall S. Clinical governance: learning and changing practice. J Fam Plann Reprod Health Care 2001; 27: 228-230.

7 Greenhalgh T. The new ethics. BMJ 2004; 328: 651 .

18 Wakley G. Clinical governance for better sexual health services. J Fam Plann Reprod Health Care 2004; 30: 260-262.

\section{Book Review}

MenoPartners: The Guy's Guide to Surviving Menopause. Christopher W Pritchard. Baltimore, MD: PublishAm: 97. Price: \$14.95 $1-4317-1760-2$

(softback)

"What a brilliant concept!" was my first response when sent this little book about the menopause, the first to be written specifically for the edification of men. With my agony aunt hat on, get many letters from women in their forties and fifties murmuring about their husbands' lack of understanding and support, and so I welcon understong and support, and so I welcomed with open arms this attempt to reach the male population

And on a first flick through the book there were many elements I applauded. The text had not fallen into the trap of using medical-speak for the lay market. The content included symptoms to inform; first-hand accounts to enable readers to sympathise; practical suggestions on how to help; a list of websites and organisations; a glossary. was particularly delighted at the statement that although the book can be bought through bookshop websites, it is also available for reading free at the author's website.

But on a more thorough reading, I started to have doubts. The author is based in the States. Hence, understandably, the style, cultural references and quoted organisations are very American indeed, making it unsuitable for the multinational market that will read it on the web Plus, the admirable disclaimer at the start of the book - that the author is going to make the book "a quick and painless as possible" - is not foll quick and painless as polible - is not fo through; the symptom lists are repeated several mes with increasingly technical vocabulary.

Most disappointingly of all, the much vaunted 'Menopartners' website, a supportive community for men and their menopausal partners, and on which the book claims to be available for free, was - at the time of writing this review - not to be found under the given URL (http://www.menopartners.net).

But maybe my negative response was jus female pickiness. I decided to test the market first hand - so handed the book over to my spouse, who is currently coping (very well) with a whe. "Patronising ... too long ... ong ...t too wordy ... these first-hand accounts seem forged to me ... wouldn't get beyond the first page."

In the end, then, I felt this book could have been shorter, more focused and less culturally specific. Crucially, it could have reliably followed through on the promised website. In short - and in the opinion of both female reviewer and male target market - it's a wonderful idea and a missed opportunity.

Reviewed by Susan Quilliam, BA, MNLP

Freelance Writer, Broadcaster and Agony Aunt, Cambridge, UK 